\title{
Management of glucose profile throughout strict COVID-19 lockdown by patients with type 1 diabetes prone to hypoglycaemia using sensor-augmented pump
}

\author{
Clara Viñals $^{1}$ (D) $\cdot$ Alex Mesa $^{1} \cdot$ Daria Roca $^{1} \cdot$ Merce Vidal $^{1} \cdot$ Irene Pueyo $^{1} \cdot$ Ignacio Conget $^{1,2,3} \cdot$ Marga Giménez $^{1,2,3}$
}

Received: 6 August 2020 / Accepted: 15 October 2020 / Published online: 30 October 2020

(c) Springer-Verlag Italia S.r.l., part of Springer Nature 2020

\begin{abstract}
Aims Spain has been one of the worst affected countries by the COVID-19 pandemic. A very strict lockdown at home was imposed with a tough restriction of mobility. We aimed to evaluate the impact of this exceptional scenario on glucose profile of patients with type 1 diabetes (T1D) prone to hypoglycaemia using sensor-augmented pump (SAP).

Methods Patients with T1D prone to hypoglycaemia using SAP (640G Medtronic-Minimed ${ }^{\circledR}$ ) for at least 6 months under the funding of a National Health Service were included in an observational, retrospective study. Data were collected in two periods: pre-lockdown (PL), February 23rd-March 7th and within lockdown (WL), April 1st to 14th 2020. The primary outcome was the difference in the proportion of time in target glucose range of $70-180 \mathrm{mg} / \mathrm{dL}$ (TIR). Additional glucometric data and total daily insulin were also analysed.

Results Fifty-nine patients were included: 33 women, age $46.17 \pm 13.0$ years and disease duration of $30.2 \pm 12.0$ years. TIR $70-180 \mathrm{mg} / \mathrm{dL}(67.6 \pm 11.8$ vs. $69.8 \pm 12.0 \%)$, time $>180(28.1 \pm 13.6$ vs. $25.5 \pm 13.1 \%)$, time $>250(6.9 \pm 6.1$ vs. $5.1 \pm 4.8)$ and estimated $\mathrm{HbA}_{1 \mathrm{c}}(6.94 \pm 0.8$ vs. $6.75 \pm 0.7 \%)$ significantly improved (PL vs. WL, respectively, $\left.p<0.05\right)$. Time in hypoglycaemia, coefficient of variation, sensor usage and total daily insulin dose remained unchanged.

Conclusions Lockdown conditions imposed by the COVID-19 pandemic may be managed successfully in terms of glycaemia control by population with DT1 prone to hypoglycaemia using SAP. The strict daily routine at home could probably explain the improvement in the time in glycemic target without increasing the time hypoglycaemia.
\end{abstract}

Keywords Type 1 diabetes $\cdot$ Hypoglycaemia $\cdot$ COVID-19 lockdown, sensor-augmented pump

\section{Introduction}

To respond to public health crisis caused by the global coronavirus disease 2019 (COVID-19) pandemic, Spain's Interior Ministry imposed a highly strict lockdown across the country in 14th March 2020, the so-called "state of alert" period [1]. This imposed drastic measures to fight the Severe acute respiratory syndrome coronavirus-2 (SARS-CoV-2), forced people to be at home modifying their daily routines becoming them very unchanging but really challenging for people with chronic diseases [2].

Appropriately managing of glucose control in type 1 diabetes (T1D) is markedly influenced by lifestyle conditions which are determined by the interplay between an individual's personal characteristics, social interactions, socioeconomic and environmental living conditions. The parameters most commonly analysed in the assessment of lifestyle influence include physical activity and nutrition, with a focus on 
monitoring carbohydrate intake [3]. However, other parameters that have to be considered include exposure to stressful life events at work and at home, sleep duration, specific unhealthy behaviours, among others $[4,5]$.

The use of continuous glucose monitoring (CGM) associated with insulin pump therapy, the so-called sensor-augmented pump (SAP) therapy, has shown a reduction in nonsevere and severe hypoglycaemia in people with T1D prone to this adverse event [6-9]. These findings lend support to the broad adoption and national health system reimbursement of this technology in the management of this high-risk population.

We wanted to take advantage of the exceptional living conditions imposed by the strict lockdown due to COVID19 pandemic crisis in Spain to evaluate the management of glucose profile by patients with T1D prone to hypoglycaemia using SAP, a critically vulnerable population with T1D.

\section{Materials and methods}

We performed an observational, retrospective study that involved reviewing the electronic medical records and databases of individuals with T1D followed at the Diabetes Unit, Endocrinology and Nutrition Department at Hospital Clínic of Barcelona. In the current analysis, patients with T1D using SAP therapy with the 640G MedtronicMinimed system (Medtronic-Minimed, Northridge, CA, USA) linked to a glucometer (Contour Next $2.4^{\circledR}$, Ascensia Diebetes Care, Parsippany, NJ, USA) and a glucose sensor (Enlite ${ }^{\circledR}$, Medtronic-Minimed, Northridge, CA, USA) for at least 6 months were included. We used anonymized CareLink-Pro ${ }^{\circledR}$ (Medtronic-Minimed, Northridge, CA, USA) data collected between two different periods: pre-COVID-19 lockdown (PL), from February 23rd to March 7th 2020 and within the COVID-19 lockdown (WL), from April 1st to April 14th 2020. The specific indication for starting SAP therapy under the funding of the Catalan National Health Service was recurrent severe and disabling hypoglycaemic episodes or hypoglycaemia unawareness despite the use of continuous subcutaneous insulin infusion (CSII).

Demographic and clinical data were recorded from computerized clinical records. Data from 14 consecutive days within the periods mentioned above were collected from uploads from each patient including CGM data using CareLink Pro ${ }^{\circledR}$ software (.csv files selecting specifically the two periods). Only downloads were included in the study if there was $>70 \%$ of CGM data in both periods.

The primary outcome of the study was defined as the difference between periods in the proportion of time spent in the target glucose range of $70-180 \mathrm{mg} / \mathrm{dL}$. In addition to this, data regarding the mean and coefficient of variation ( $\leq$ and $>36 \%$ ) of sensor-measured glucose concentrations over both periods, $\%$ time with glucose concentrations in hypoglycaemia ( $<70 \mathrm{mg} / \mathrm{dL} ;<54 \mathrm{mg} / \mathrm{dL})$ and hyperglycaemia ( $>180 \mathrm{mg} / \mathrm{dL} ;>250 \mathrm{mg} / \mathrm{dL}$ ), estimated $\mathrm{HbA}_{1 \mathrm{c}}$ $\left(\mathrm{eHbA}_{1 \mathrm{c}}\right)$, mean sensor glucose and standard deviation of sensor glucose were also obtained. Information regarding SAP settings and usage was obtained from downloads: total insulin dose per day, total basal insulin and bolus insulin per day, number of bolus per day, predictive low glucose suspend (PLGS) target, sensor use, time suspension on low per day, time suspension before low per day, total insulin suspensions per day and number of low, high alarms per day and total carbohydrate consumption per day were recorded. The study have been reviewed by the local ethics committee (HCB/2020/0601) and have therefore been performed in accordance with the ethical standards laid down in an appropriate version of the 1964 Declaration of Helsinki. All subjects gave informed consent.

\section{Statistical analysis}

We planned a study of a continuous response variable from pairs of study subjects. The power calculation was done for the primary outcome if the true difference in the mean response of matched pairs is 2.50 , with a standard deviation of 6.50 , we will need to study 56 pairs of subjects to be able to reject the null hypothesis that this response difference is zero with probability (power) 0.80 . The Type I error probability associated with this test of this null hypothesis is 0.05 .

The results are presented as mean \pm SD or proportions. Comparisons between continuous variables were performed using a paired Student's $t$ test. Comparisons between categorical variables were performed using a McNemar's test. A $P$-value $<0.05$ was considered statistically significant. Data analysis was carried out with SPSS software, version 25.0 (IBM SPSS Statistics, Armonk, NY).

\section{Results}

Fifty-nine patients were included: 33 women, full population characteristics are described in Table 1. Regarding nephropathy 3 had microalbuminuria, 2 macroalbuminuria, none of them were on dialysis. None of the patients included were pregnant, 2 were on pre-pregnancy care. All of them were treated with SAP therapy.

Regarding glucometric results, during the lockdown patients achieved more time in range $(70-180 \mathrm{mg} / \mathrm{dL})$, less time in hyperglycaemia ( $>180 \mathrm{mg} / \mathrm{dL}$ and $>250 \mathrm{mg} / \mathrm{dL}$ ), a better $\mathrm{eHbA}_{1 \mathrm{c}}$ without increasing time spent in hypoglycae$\mathrm{mia}(<70 \mathrm{mg} / \mathrm{dL}$ and $<54 \mathrm{mg} / \mathrm{dL})$. There was no difference in sensor use between both periods (Table 2).

The proportion of patients achieving consensus statement targets [10] in relation to sensor glucose values in 
Table 1 Basal characteristics

\begin{tabular}{ll}
\hline$N=59$ & \\
\hline Gender (male/female) & $26 / 33(44.1 / 55.9)$ \\
Age (years) & $46.18 \pm 13.02$ \\
Evolution T1D (years) & $30.24 \pm 11.98$ \\
Ethnicity Caucasian & $59(100)$ \\
Diabetic Complications & $34(57.6)$ \\
Retinopathy & $33(55.9)$ \\
Nephropathy & $5(8.6)$ \\
Cardiovascular disease & $3(5.1)$ \\
\hline
\end{tabular}

Data expressed as number (percentage) or mean \pm standard deviation T1D type 1 diabetes

both periods was analysed (Table 3). Although there was a tendency to an improvement in glucose exposure comparisons favouring the lockdown period, all of these differences were not statistically significant, with the exception of $\mathrm{eHbA}_{1 \mathrm{c}}<7 \%$ (48.2\% vs. $58.9 \%$, during PL and WL, respectively, $p=0.03)$.

In the lockdown period, a $19.6 \%(n=11)$ of the subjects achieved an improvement $\geq 0.4 \%$ in $\mathrm{eHbA}_{1 \mathrm{c}}$ and a $33.3 \%$ $(n=19)$ shown an improvement $\geq 5 \%$ in time in range (between 70 and $180 \mathrm{mg} / \mathrm{dl}$ ). These differences could be considered clinically relevant.

Regarding SAP usage and settings information, there was no difference in total daily insulin dose $(41.34 \pm 17.34$ vs. $40.83 \pm 19.47 \mathrm{IU} /$ day), total basal insulin per day, total bolus insulin per day, number of bolus per day, total carbohydrate consumption $(13.83 \pm 6.79$ vs. $12.82 \pm 6.33$ exchanges-10 g each/day), predictive low glucose suspends (PLGS) targets, time suspensions on low per day, time suspensions before low per day and total insulin suspensions, comparing PL and WL periods. During the WL, there was a decrease in the number of high alarms per day $(4.59 \pm 3.17$ vs. $3.87 \pm 2.75 ; p<0.001)$.
Table 3 Proportion of patients achieving consensus statements in relation to sensor glucose values during the pre-lockdown and within the lockdown period

\begin{tabular}{llll}
\hline & Pre-lockdown & Within-lockdown & $p$ \\
\hline$<1 \%$ of time $<54 \mathrm{mg} / \mathrm{dl}$ & $37(64.9)$ & $36(63.2)$ & 1.0 \\
$<4 \%$ of time $<70 \mathrm{mg} / \mathrm{dl}$ & $32(56.1)$ & $32(56.1)$ & 1.0 \\
$>70 \%$ of time $70-180 \mathrm{mg} /$ & $26(45.6)$ & $29(50.9)$ & 0.549 \\
$\quad \mathrm{dl}$ & & & \\
$<25 \%$ of time $>180 \mathrm{mg} / \mathrm{dl}$ & $24(42.1)$ & $27(47.4)$ & 0.453 \\
$<5 \%$ of time $>250 \mathrm{mg} / \mathrm{dl}$ & $28(49.1)$ & $33(57.9)$ & 0.227 \\
$<7 \%$ eHbA & $27(48.2)$ & $33(58.9)$ & 0.031 \\
$<36 \%$ of $\mathrm{CV}$ & $33(57.9)$ & $31(54.4)$ & 0.804 \\
\hline
\end{tabular}

Data expressed as number (percentage)

$e H b A_{l c}$ estimated $\mathrm{HbA}_{1 \mathrm{c}}, C V$ coefficient of variation

Through both periods of time, there were not clinically significant acute hypo or hyperglycaemic complications and none of the patients needed hospitalization.

\section{Discussion}

The use of SAP allows patients with T1D prone to hypoglycaemia to effectively and safely manage glucose control even throughout the exceptional living conditions imposed by the lockdown due to COVID-19 crisis. Lockdown period was associated with some improvement in glucometric parameters especially those associated with glucose exposure without increasing the risk of hypoglycaemia.

The COVID-19 pandemic has been a challenge for worldwide population, but fortunately, it has rushed the need for telemedicine and virtual diabetes clinic to complement standard outpatient care, this being especially suitable in patients with T1D using advanced treatments including CGM and SAP [11, 12].

Due of the rapid spread of COVID-19 worldwide public authorities had to impose extraordinary policies like lockdowns to reduce the risk of infection to the population. The
Table 2 Comparison of glycemic control during the pre-lockdown and within the lockdown period

\begin{tabular}{lllll}
\hline & Pre-lockdown & Within-lockdown & Mean difference response & $p$ \\
\hline Mean sensor glucose (mg/dl) & $153.23 \pm 22.36$ & $147.84 \pm 19.54$ & $-5.39 \pm 10.45$ & $<0.001$ \\
Estimated $\mathrm{HbA}_{1 \mathrm{c}}(\%)$ & $6.94 \pm 0.78$ & $6.75 \pm 0.71$ & $-0.19 \pm 0.35$ & $<0.001$ \\
Time $<54 \mathrm{mg} / \mathrm{dl}(\%)$ & $1.21 \pm 1.39$ & $1.44 \pm 1.97$ & $0.023 \pm 1.24$ & 0.174 \\
Time $<70 \mathrm{mg} / \mathrm{dl}(\%)$ & $4.34 \pm 3.32$ & $4.67 \pm 3.88$ & $0.11 \pm 1.58$ & 0.305 \\
Time $70-180 \mathrm{mg} / \mathrm{dl} \mathrm{( \% )}$ & $67.58 \pm 11.77$ & $69.78 \pm 12.07$ & $2.20 \pm 6.77$ & 0.017 \\
Time $>180 \mathrm{mg} / \mathrm{dl}(\%)$ & $28.08 \pm 13.59$ & $25.54 \pm 13.13$ & $-0.77 \pm 4.86$ & 0.007 \\
Time $>250 \mathrm{mg} / \mathrm{dl}(\%)$ & $6.89 \pm 6.12$ & $5.13 \pm 4.75$ & $-1.76 \pm 3.68$ & 0.001 \\
Coefficient of variation $(\%)$ & $35.71 \pm 3.26$ & $35.35 \pm 4.56$ & $-0.36 \pm 3.82$ & 0.479 \\
Sensor use $(\%)$ & $84.83 \pm 9.70$ & $85.02 \pm 10.76$ & $0.13 \pm 9.00$ & 0.874 \\
\hline
\end{tabular}

Data expressed as mean \pm standard deviation or number (percentage) 
effect of lockdown in glycemic control in people with T1D was totally unknown; however, it has been recently assessed in a population with T1D using CGM and hybrid closedloop systems (HCL). Bonora et al. [13] described 33 individuals with T1D using CGM who improved TIR from 54.4 to $65.2 \%$ during the lockdown in those who stayed at home, with no changes in those who kept working. Tornese et al. [14] reported 13 adolescents treated with an HCL system and good glycemic control at baseline (TIR 68\%), in which glycemic control improved $(+3 \%)$ especially in those who continued physical activity during the lockdown. Recently, Beato [15] has described the effects of the lockdown in a Spanish population, showing no deleterious effect of the lockdown in a cohort of patients with T1D treated with multiple insulin injections and CGM. Recently, it has been claimed that the improvement in glucose control observed during lockdown in a T1D population treated with CSII or multiple daily injections could be due to more stable rhythm of life and regular mealtimes [16, 17].

The use of SAP has been significantly associated with a reduction in the frequency of non-severe and severe hypoglycaemic events in hypoglycaemia-prone adults compared to the use of CSII without CGM [6]. We evaluated the impact of lockdown in a different and particularly vulnerable population. We included people with T1D prone to recurrent severe hypoglycaemia. In comparison with the population included in previous manuscripts [13-17], the percentage of time $<54 \mathrm{mg} / \mathrm{dL}$ in our population is almost double before lockdown. Despite this, the percentage of TIR during the lockdown and the improvement observed in glucose exposure in our study was much higher without any significant increase in hypoglycaemia. Close to $60 \%$ of our patients reached $<4 \%$ of time $<70 \mathrm{mg} / \mathrm{dL}, 50 \%$ had sensor glucose values within $>70 \%$ of TIR and almost $60 \%$ of them had an $\mathrm{eHbA}_{1 \mathrm{c}}<7 \%$. Thus, regular and homogeneous daily life conditions and activity in a safe home environment imposed by strict lockdown may contribute to facilitate glucose management also in a high-risk population with T1D. This includes increased consumption of homemade food facilitating carbohydrate counting and the administration of boluses at time, decreased workloads and increased time to cope with diabetes.

Recently, we have published [18] the impact of COVID19 lockdown in a T1D population prone to hypoglycaemia using standalone CGM. We have found that this high-risk population managed successfully in terms of glycemic control. In this occasion, we include a different subpopulation of patients, all of them treated with SAP therapy. The effectiveness to achieve glycemic targets may be different using SAP therapy compared to standalone CGM. In fact, this can be easily recognized in the results of glucose targets in both studies. In this context, the success of both therapies during lockdown warrants a specific analysis.
Actually, we had the opportunity to analyse SAP usage and settings information to explain the differences between the two periods of time (PL vs. WL), unfortunately, no significant differences were seen in any of the available parameters.

Our results contrast with the estimation of the effects of lockdown due to COVID-19 in glycemic control published by Ghosal et al. [19]. In that study, the authors estimated a worsening of glycemic control related to the duration of lockdown, as well as, an increase in the diabetic complication rates.

The greatest differences in glycemic control were seen in time above $180 \mathrm{mg} / \mathrm{dl}$ and $250 \mathrm{mg} / \mathrm{dl}$ rather than TIR. This is in line with a recent publication [20] suggesting that time above $180 \mathrm{mg} / \mathrm{dl}$ could be a better index of overall quality of glucose control than is TIR.

There are limitations in our study. First of all, it is a retrospective, observational real-world study and due to this nature only an association between SAP data obtained in both periods of time, and no causation, can be inferred from our results. The glucose profile information was obtained from two short periods of time, so we cannot know if the positive results could be extended in time. It has to be considered that we included a highly selected T1D population using an advanced therapy. As such, their engagement in the management of their disease is likely to be different to other T1D patients, and this limit the extrapolation of the results. Although we analysed SAP in this study, variations in daily physical activity and meal composition which may influence glucose control were not investigated. Finally, the caucasian population included in our study belongs to a single reference Diabetes Unit in Spain with a high expertise in the management of SAP, thus the findings could not be considered representative for other populations treated in different clinics and countries. On the other hand, our study has some strength. The exceptional, almost experimental, living conditions imposed by the very strict lockdown measures imposed in Spain gave to us the opportunity to test the effects of restrictive and homogenous lifestyle conditions staying at home on glycemic profile of patients with T1D. We could also evaluate the effectiveness of SAP technology with advanced features in a particular group of high-risk patients in such conditions. Finally, because we preplanned the sample size of subjects in our study, we feel confident with the difference found in TIR between both periods of time. A $2.2 \%$ increase in TIR (30 min per day) and a $0.2 \%$ decrease in eHbA $1_{c}$, if maintained, could be considered clinically relevant. Finally, because we preplanned the sample size of subjects in our study, we feel confident with the difference we found in TIR between both periods of time. 


\section{Conclusion}

In summary, very restrictive lockdown conditions imposed by the COVID-19 pandemic may be managed successfully in terms of glycemic control by population with T1D prone to hypoglycaemia using advanced SAP. The strict daily routine at home could probably explain the improvement in the time in glycemic target without increasing the time hypoglycaemia.

Acknowledgements We would like to thank the altruistic participation of the patients.

Author's contributions $\mathrm{CV}$, MG and IC contributed to the study concept, designed the study and provided statistical advice on study design. $\mathrm{CV}$, IC had full access to all data in the study and takes responsibility for the integrity of data and accuracy of data analysis and wrote the article. AM, DR, IP, MV collected and reviewed the data. All authors contributed to the interpretation of data and the drafting of the report. They critically revised the report for important intellectual content and approved the version to be published.

Funding None.

\section{Compliance with ethical standards}

Conflict of interest Dr Viñals has received lecturing fees from NovoNordisk A/S, Medtronic Inc., Sanofi-Aventis and MSD. Dr Giménez has received lecturing and consulting fees from Medtronic Inc., Eli Lilly \& Co., NovoNordisk A/S, Sanofi-Aventis, Astra Zeneca and MSD. Dr Conget reported receiving lecturing and consulting fees from Medtronic Inc., Bayer AG, GlaxoSmithKline, Eli Lilly \& Co., NovoNordisk A/S, Sanofi-Aventis, Novartis, Astra Zeneca and MSD.

Ethical standard The study have been reviewed by the local ethics committee (HCB/2020/0601) and have therefore been performed in accordance with the ethical standards laid down in an appropriate version of the 1964 Declaration of Helsinki.

Informed consent All subjects gave informed consent.

\section{References}

1. La Presidencia M DE, Con Las Cortes Igualdad RE: I. Disposiciones Generales Ministerio de la Presidencia, Relaciones con las Cortes e Igualdad, 2020. BOE-A-2020-3692. https://www. boe.es/eli/es/rd/2020/03/14/463. Accessed 1 June 2020

2. Chu DK, Akl EA, Duda S et al (2020) Physical distancing, face masks, and eye protection to prevent person-to-person transmission of SARS-CoV-2 and COVID-19: a systematic review and meta-analysis. Lancet. https://doi.org/10.1016/S0140 $-6736(20) 31142-9$

3. Association AD (2020) 5. Facilitating behavior change and well-being to improve health outcomes: standards of medical care in diabetes-2020. Diabetes Care 43:S48-S65. https://doi. org/10.2337/dc20-S005

4. Hansen UM, Skinner T, Olesen K, Willaing I (2019) Diabetes distress, intentional hyperglycemia at work, and glycemic control among workers with type 1 diabetes. Diabetes Care 42:797-803. https://doi.org/10.2337/dc18-1426
5. Griggs S, Redeker NS, Grey M (2019) Sleep characteristics in young adults with type 1 diabetes. Diabetes Res Clin Pract 150:17-26. https://doi.org/10.1016/j.diabres.2019.02.012

6. Bosi E, Choudhary P, de Valk HW et al (2019) Efficacy and safety of suspend-before-low insulin pump technology in hypoglycaemia-prone adults with type 1 diabetes (SMILE): an openlabel randomised controlled trial. Lancet Diabetes Endocrinol 7:462-472. https://doi.org/10.1016/S2213-8587(19)30150-0

7. Ly TT, Nicholas JA, Retterath A et al (2013) Effect of sensoraugmented insulin pump therapy and automated insulin suspension vs standard insulin pump therapy on hypoglycemia in patients with type 1 diabetes: a randomized clinical trial. JAMA 310:1240-1247. https://doi.org/10.1001/jama.2013.277818

8. Beato-Víbora PI, Quirós-López C, Lázaro-Martín L et al (2018) Impact of sensor-augmented pump therapy with predictive low-glucose suspend function on glycemic control and patient satisfaction in adults and children with type 1 diabetes. Diabetes Technol Ther 20:738-743. https://doi.org/10.1089/ dia.2018.0199

9. Abraham MB, Nicholas JA, Smith GJ et al (2018) Reduction in hypoglycemia with the predictive low-glucose management system: a long-term randomized controlled trial in adolescents with type 1 diabetes. Diabetes Care 41:303-310. https://doi. org/10.2337/dc17-1604

10. Battelino T, Danne T, Bergenstal RM et al (2019) Clinical targets for continuous glucose monitoring data interpretation: recommendations from the international consensus on time in range. Diabetes Care 42:1593-1603. https://doi.org/10.2337/ dci19-0028

11. Keesara S, Jonas A, Schulman K (2020) Covid-19 and health care's digital revolution. N Engl J Med. https://doi.org/10.1056/ NEJMp2005835

12. Nørgaard K (2020) Telemedicine consultations and diabetes technology during COVID-19. J Diabetes Sci Technol. https:// doi.org/10.1177/1932296820929378

13. Bonora BM, Boscari F, Avogaro A et al (2020) Glycaemic control among people with type 1 diabetes during lockdown for the SARS-CoV-2 outbreak in Italy. Diabetes Ther. https://doi. org/10.1007/s13300-020-00829-7

14. Tornese G, Ceconi V, Monasta L et al (2020) Glycemic control in type 1 diabetes mellitus during COVID-19 quarantine and the role of in-home physical activity. Diabetes Technol Ther. https ://doi.org/10.1089/dia.2020.0169

15. Beato-Víbora PI (2020) No deleterious effect of lockdown due to COVID-19 pandemic on glycaemic control, measured by glucose monitoring, in adults with type 1 diabetes. Diabetes Technol Ther. https://doi.org/10.1089/dia.2020.0184

16. Capaldo B, Annuzzi G, Creanza A et al (2020) Blood glucose control during lockdown for COVID-19: CGM metrics in Italian adults with type 1 diabetes. Diabetes Care 43:e88-e89. https:// doi.org/10.2337/dc20-1127

17. Fernández E, Cortazar A, Bellido V (2020) Impact of COVID19 lockdown on glycemic control in patients with type 1 diabetes. Diabetes Res Clin Pract 166:108348. https://doi. org/10.1016/j.diabres.2020.108348

18. Mesa A, Viñals C, Pueyo I et al (2020) The impact of strict COVID-19 lockdown in Spain on glycemic profiles in patients with type 1 diabetes prone to hypoglycemia using standalone continuous glucose monitoring. Diabetes Res Clin Pract. https ://doi.org/10.1016/j.diabres.2020.108354

19. Ghosal S, Sinha B, Majumder M, Misra A (2020) Estimation of effects of nationwide lockdown for containing coronavirus infection on worsening of glycosylated haemoglobin and increase in diabetes-related complications: a simulation model using multivariate regression analysis. Diabetes Metab 
Syndr Clin Res Rev 14:319-323. https://doi.org/10.1016/j. dsx.2020.03.014

20. Rodbard D (2020) Glucose time in range, time above range, and time below range depend on mean or median glucose or HbA1c, glucose coefficient of variation, and shape of the glucose distribution. Diabetes Technol Ther 22:492-500. https:// doi.org/10.1089/dia.2019.0440
Publisher's Note Springer Nature remains neutral with regard to jurisdictional claims in published maps and institutional affiliations. 